\title{
Information management in Malawi's prevention of Mother-to-Child Transmission (PMTCT) Program: Health workers' perspectives
}

\section{Wiza Kumwenda ${ }^{1,3}$, Gregory Kunyenje ${ }^{1}$, Jimmie Gama ${ }^{1,2}$, Jacqueline Chinkonde ${ }^{4}$, Francis Martinson ${ }^{3}$, Irving Hoffman ${ }^{3}$, Mina Hosseinipour $^{3}$, Nora Rosenberg ${ }^{3}$}

1.University of Malawi, Chancellor College, Malawi 2.University of Malawi, College of Medicine, Malawi 3.UNC Project, Malawi

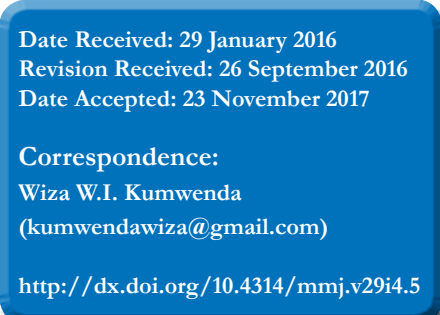

\begin{abstract}
Aim
Abstract

Health workers are the key drivers for strengthening Prevention of Mother-to-Child Transmission (PMTCT) program information management in the health facilities. Thus understanding how health workers perceive information management can enlighten areas that require interventions to improve information management processes in the health facilities. The purpose is to assess health workers' perceptions toward PMTCT program information management and factors affecting information management in the health facilities.

Methods

The study was conducted in five out of forty-three health facilities providing PMTCT services in Lilongwe district and thirty out of sixty-eight health workers were recruited across the study sites. Purposive and convenience sampling were used. Semi-structured questionnaires and in-depth interviews were used to collect demographic information and health workers' perceptions toward information management, respectively. Thematic and content analysis techniques were employed for qualitative data, while descriptive statistics were used for quantitative data.

Results

Most health workers perceived information management tasks as part of their job description, but less important to provision of clinical services. For many, use of information technology tools was viewed as beneficial and valuable, whereas the paper-based system was perceived as tedious and difficult to manage. In addition, some believed lack of feedback, information sharing, and poor attitude toward information management tasks were challenges.

Conclusion

Based on the study findings, there is need to find ways of motivating data quality improvement practises in the health facilities, as health workers view this as a tangential, non-essential part of their job. Health facility leadership needs to promote an information culture through enforcement of meetings, supervision and provision of feedback. The government and its partners should continue rolling out and enhancing competence of health workers on EMR in the health facilities whilst also addressing challenges mentioned in the study.
\end{abstract}

\section{Introduction}

Prevention of Mother-to-Child Transmission (PMTCT) is a set of clinical approaches for preventing the transmission of Human Immunodeficiency Virus (HIV) from an infected mother to her child. Effective PMTCT efforts drastically reduce paediatric HIV infection. They also serve as an entry point to care, treatment and support for HIV infected women and their exposed children and families ${ }^{1}$. Collection, recording and use of high-quality routine data on these services and outcomes for HIV-positive mothers and HIVexposed infants is not only essential for monitoring and evaluation of PMTCT programs, but also paramount in the clinical management of patients and in managing stocks of HIV test kits and drugs ${ }^{2}$.

In Malawi, women of reproductive age carry a high burden of HIV infection. The government of Malawi initiated PMTCT programs in 20013. In Lilongwe district, implementation of PMTCT services started in April 2002 with 4 health facilities ${ }^{4}$. The coverage of PMTCT services has expanded rapidly and initiatives to improve delivery of PMTC'T services have been equally impressive. There are more than 40 sites in Lilongwe, 130 sites in the central west zone, and over 700 sites nationally with PMTCT Services ${ }^{5,6}$. Malawi has recently developed Option B+, a lifelong ART program for pregnant and breastfeeding women regardless of CD4 cell counts, in response to the specific needs of its people and available health infrastructure ${ }^{7}$

With the scale-up of Option B+ PMTCT activities, there is the need to ensure good quality information is being produced from the health facilities. Since health facilities are the established base for collecting, processing, and reporting routine primary data in PMTCT program. The data produced at health facility level is used at district, zonal and national level for monitoring and measuring the performance, ordering commodities, deploying staff, and informing decision-making of the national PMTCT program ${ }^{3}$.

Malawi government has put in place mechanisms to ensure that information produced in health facilities is of good quality by simplifying and standardizing data collection and reporting tools, providing training on the tools, and conducting routine quarterly site supervision, and clinical mentorship ${ }^{6,8}$. Additionally, they have implemented electronic data systems in some health facilities with mechanisms to improve data quality, such as numerical validation, bounds checking and alerts ${ }^{9,10}$. Despite efforts to improve the PMTCT routine health information system performance in the health facilities, information produced is still considered to be unreliable, incomplete, and of low quality ${ }^{11,12}$. For example, records continue to have missing and inaccurate information ${ }^{13}$. Thus, there is need to strengthen data quality 
improvement practices in PMTCT Information Management (IM) at facility level. This can be achieved by understanding how people behave when performing IM activities.

Health workers are the key drivers for quality improvement practice. To ensure that data is relevant for quality improvement practice, it must serve the interests of those who have to collect it ${ }^{14-16}$. Thus to understand health worker interests there is need to assess how health workers perceive IM activities ${ }^{17}$. In the context of PMTCT program, health workers perceptions toward information management are unknown.

This study investigates how health workers perceive PMTCT IM and which areas they consider to be affecting PMTCT IM in the health facilities. The objectives of this study were two-fold: 1) to explore and describe the perceptions of the health workers regarding PMTCT data, and 2) to explore health workers' perceived gaps and challenges in PMTCT IM at the health facility.

\section{Methods}

\section{Study design, setting and population}

A cross-sectional mixed method study was conducted between July and November 2011 across five of the fortythree health facilities that were providing PMTCT services in the Lilongwe district of Malawi: Bwaila District Hospital, Area 18, Kawale, Nathenje, and Chitedze Health Centres. The study sites were among the first sites to implement PMTCT services in Lilongwe district and they also reflect a broad spectrum of healthcare delivery. These facilities are high volume sites; they serve approximately 500 PMTCT patients per quarter. Sites were also selected based on proximity.

The study mainly used qualitative approach and was complemented by minor quantitative approach. 30 out of the 68 health workers were recruited across 5 health facilities. The process of recruitment at each study site was initiated by purposively identifying and enrolling the lead health worker on PMTCT activities who then helped in recruiting other health workers. The health workers (nurses, counsellors, clinicians, and data personnel) were eligible, if they were available on the days the researcher was at these sites and were willing to participate in the study. Of the 36 health workers that were approached, 5 were unavailable and one refused participation.

\section{Ethical consideration}

Ethical approvals were obtained from Malawi National Health Science Research Committee. Only academic approvals were obtained from University of Malawi-Chancellor College. The participants provided informed consent. Data for analysis did not include any personal identifiers and were analyzed anonymously. The participants were told not to mention their names during recorded in-depth interviews. Data were stored on secure, encrypted password-protected servers.

\section{Data collection and analysis}

Data were collected through observation, semi-structured questionnaire and in-depth interviews.The questions included demographics, health workers' role in PMTCT IM, tools used in PMTCT IM activities, perceived usefulness of PMTCT IM, challenges affecting PMTCT IM activities and the proposed solutions. Observation was used for review of the information management systems. The questionnaire was first administered. This was later complemented by face to face recorded in-depth interviews. The interviews were conducted in either English or Chichewa or using both languages depending on participant preference. Each interview took approximately 40-60 minutes.

Qualitative data were analysed using thematic and content techniques. The first step was that the audio-recorded interviews were translated, when necessary, and transcribed into English by a transcriber. Then the principal investigator listened and read all transcripts at least twice to confirm their accuracy. This led to the development of codes and a code book. In the second phase he coded all transcripts and updated the code book, when necessary. The third step was to identify emerging themes from the data. The last step was to interpret the data. A social scientist reviewed the transcripts, codes, themes, and interpretation for validation and did not find any issue.

Quantitative analysis of structured questions was conducted using SPSS statistical software using descriptive statistics.

\section{Results}

\section{Characteristics of study participants}

30 participants were enrolled and $80 \%$ were female. The median age of study participants was 34 years (25-65 years) and the mean age was 37 years (standard deviation $=10.5$ years). The majority of respondents had secondary education $(\mathrm{N}=20,66 \%)$ and some had tertiary education $(\mathrm{N}=10,33 \%)$. Job cadres included nurses $(\mathrm{N}=21,70 \%)$, counselors $(\mathrm{N}=4$, $13 \%)$, data clerks $(\mathrm{N}=2,7 \%)$, data officers $(\mathrm{N}=2,7 \%)$, and a clinical officer $(\mathrm{N}=1,3 \%)$. Bwaila Hospital had the largest number of study participants with $33 \%$ (10 of 30) whilst the rest of study sites each had $17 \%$ (5 of 30 ) of study participants.

\section{Perception on PMTCT IM tools}

Management of PMTCT information across the study sites was primarily paper-based and complemented, in some instances with IT tools. Paper-based tools included antenatal, maternity, and HTC registers, health passports, mastercards, and reporting forms.

IT tools allows health workers to collect, store retrieve, communicate, and transfer PMTCT information electronically in the health facility. The following were IT tools being used: touch screen electronic medical records (EMR) used for data collection and reporting, computers for general use, personal and health system cell phone for tracing participants with missed visits, and wireless message devices for communication between facilities or with the District Health Office.

The main IT tool employed as a strategy by government to improve performance of information management in the health facilities was EMR. This tool aimed at improving the quality of PMTCT service delivery through efficient information management ${ }^{18}$. The tool was being piloted hence they had to run it parallel with paper based system.

Table 1 illustrates the distribution of IT tools implemented across the facilities and their functionality status:

\section{Table 1: Technologies}

\begin{tabular}{|c|c|c|c|c|c|c|c|c|}
\hline Health & \multicolumn{2}{|c|}{ Touch Screen } & \multicolumn{2}{|c|}{ Computer System } & \multicolumn{2}{|c|}{$\begin{array}{l}\text { Wireless } \\
\text { Mess a ge } \\
\text { devices }\end{array}$} & Cell phone & \\
\hline Facility & A & $\mathrm{F}$ & A & $\mathrm{F}$ & A & $\mathrm{F}$ & A & $\mathrm{F}$ \\
\hline \multicolumn{9}{|c|}{ B w a il a } \\
\hline Hospital & $v$ & $v$ & $v$ & $v$ & $v$ & $x$ & $v$ & $v$ \\
\hline Nathenje & $\mathrm{x}$ & $n / a$ & $v$ & $v$ & $v$ & $v$ & $v$ & $v$ \\
\hline Area 18 & $x$ & $n / a$ & $v$ & $\mathrm{x}$ & $v$ & $x$ & $v$ & $v$ \\
\hline Kawale & $\mathrm{x}$ & $n / a$ & $\mathrm{x}$ & $\mathrm{n} / \mathrm{a}$ & $v$ & $\mathrm{x}$ & $v$ & $v$ \\
\hline Chitedze & $x$ & $n / a$ & $\mathrm{x}$ & $n / a$ & $v$ & $x$ & $v$ & $v$ \\
\hline
\end{tabular}

Many health workers had a negative perception toward paper based system compared to electronic medical records or computer systems that were perceived to be efficient 
and effective. Paper-based tools were thought to be time consuming, difficult to manage, not easy to use and expensive.

"Yes, because it [the computer-based system] is cheaper than using plain paper or stationery... am able to retrieve the information about the client." (Data Clerk)

"There is lack of information technology infrastructure which results in using an outdated way of processing and keeping data (hard copy) which is easily lost or damaged." (Data Officer)

Other participants had reservation toward the usefulness of IT tools especially computers because they had limited access, inadequate knowledge or skill set.

"We had a computer which has been down for 3 months now. It was used for producing reports. However, I have never used it because I don't know how". (Nurse)

"No the computer is not working for 2 years, I don't know, it was not useful because it was not open for everyone as I could see it was for the big men [superiors]... In future computers are required." (Counselor)

Nonetheless, all the respondents indicated that they had the intention to use IT solutions in future, if the mentioned limiting factors were to be addressed.

\section{Perception on PMTCT Information Management tasks}

PMTCT programmatic data management is performed at each point where PMTCT clinical care is provided within the health facilities (HTC, antenatal, maternity, and postnatal). These tasks are performed by a diverse of cadres (nurses, data personnel, counsellors, clinician), starting from patient registration by data personnel to discharge by nurse. Thus for the study all the cadres involved in PMTCT IM tasks were classified as health worker. However, it was noted that nurses were considered as core/lead personnel for PMTCT activities.

Participants identified data compilation (97\%), data quality assurance or control (83\%), data documentation (97\%), and report development $(100 \%)$ as PMTCT IM tasks being performed in the health facilities. The tasks were recognized as part of their job description and were considered vital:

"When you are a PMTCT provider you are supposed to collect the data as part of your job. The information that you collect is right since you have the understanding of what has been done." (Nurse)

"In nursing, we believe in documentation. Documentation involves data collection. So, you feel like, information not recorded is work not done." (Nurse)

Although health workers recognized IM tasks to be part of their job, they considered clinical service provision as their primary role. Among nurse participants, $71.4 \%$ indicated that information management tasks were secondary to clinical services.

"Yes, it is relevant, but it's too tiresome. As a nurse, my duty is to provide PMTCT services not collecting data." Nurse

"It is better to provide PMTCT services to a client than documenting." (Nurse)

Performing information management tasks was also viewed to be tiring, tedious, and time consuming.

"We work, process data and then put it in the registers. It's hectic because, most times, by the time we finish helping patients we are too tired to compile data in the registry. This is why we rely on the clerks to help us. However, they fail to do all the work. So, at the end of the month, it's still us working on the data as we compile reports to submit to the DHO." (Nurse)

"It is time consuming. For example, there is shortage of health workers bere...so, one finds bimself or herself doing everything [PMTCT activities $7 . .$. and hmm, recording PMTCT data is time consuming because of these registers." (Nurse)

\section{Perceived uses of PMTCT information}

Health workers perceived PMTCT information to be useful because it was being used for clinical care, planning, evidence of work completion, and reporting.

"Yes. It is very useful because at the end of the day, you know how many people are coming to the clinic, and how many are infected and bow many receive PMTCT education." (Nurse)

"Yes, Management should know how many patients visit this clinic per day or per month... so that they should not have problems in budgeting or knowing what has been mismanaged." (Data Clerk)

The majority of the respondents emphasized data usage as a means of obtaining new stock, as evidence that they are working, or to produce reports for their supervisors.

"PMTCT information is useful for supervisors because they are able to know that I have worked, so PMTCT information that I manage act as my evidence." (Nurse)

\section{Challenges of PMTCT information management system}

Participants reported several factors that hindered PMTCT IM systems in the health facilities including lack of feedback, training, teamwork, negative attitude toward information management tasks, and high workload. The majority of the study participants $(80 \%)$ reported that they had not received feedback on the submitted PMTCT reports and or queries raised that required feedback.

"If we request for feedback from nurses they just say we have sent the information to the DHO but nothing happens." (Counsellor)

The feedback process was perceived as fundamental for quality improvement practises and a motivational factor for health workers to perform well.

"Ah as of me, I have never received feedback from the DHO. However, feedback is useful because sometimes you correct or make things better when you bave been told that you did not do well in a particular area, you keep that in mind and, next time, you make sure that the report produced is better." (Nurse)

Inadequate training was mentioned as another contributing factor to IM underperformance in the health facilities. Only $36.7 \%$ of the participants indicated to have attended PMTCT training with IM component and $20 \%$ to have attended refresher training in the previous 12 months. Those who have not attended the trainings are supposed to be briefed by those who do receive training. However, sometimes attendees fail to clearly impart training proceedings or neglect to brief others at all on what they have learned.

"...like filling in of these registers, only two went for a training workshop and the rest were just briefed. So many mistakes were made in these books last month." (Nurse)

"Frequent change of reporting format occurs even before we are conversant with the current ones and sometimes orientation is not done" (Nurse)

Participants also mentioned that high workload and lack of sharing resources contributed to underperformance of PMTCT information management activities.

"T have never read the PMTCT guidelines because they are kept with the nurses who do not share" Counsellor

"The problem is that there is shortage of health workers most of the time there can be one nurse doing all the things from antenatal, maternity so it's a challenge." (Nurse)

Participants recognized their own and their colleagues' attitudes as contributing to underperformance of the PMTCT IM system. Health workers were reluctant to resolve information management issues.

"In meetings, we discuss about documentation. The cause of poor documentation is laziness because when you ask them [PMTCT colleagues] whether they have corrected their documentation mistakes, they just say ob! I just forgot or I was busy, though they know bow to 


\section{do it." (Nurse)}

They even recognised that their attitude toward IM processes contributed to the underperformance. The majority of health workers suggested that there was need to change attitude in order for PMTCT IM system to perform.

"Health workers attitude should change and see that information management is everyone's responsibility." (Nurse)

Participants recommended that there should be increased supervision, team meetings, improved feedback, increased trainings, and recruitment of data clerks to improve performance of PMTCT IM system in the health facilities.

"There should be like supported supervision on how the services are being provided at the facility and that supported supervision should be from top management." (Nurse)

"I think that we should be having monthly meeting on HMIS." (Nurse)

\section{Discussion}

Our study has focused on understanding how front-line implementers of Malawi's PMTCT program perceive information management. While health workers recognize the importance of information management, they consider these tasks to be time-consuming secondary roles that distract from their primary role of care delivery.

Our primary finding that information management is perceived as a distraction is not unique to this setting. Other research has indicated that health workers see information management as a burden, and as a result give it second priority ${ }^{19-21}$. This perception reflects a culture where information is not valued as integral to patient care. It also contributes to poor information management practice, which can ultimately lead to poor patient outcomes, as the health system cannot adequately order commodities or deploy staff thus impeding health information system goal which is aimed at improving data quality and decision making ${ }^{22}$.

Although PMTCT program data in the health facilities is perceived as potentially useful, utilization of this data is limited. One general perception of these health workers is that data usage is for ordering stock, something that other studies have indicated as well ${ }^{15,21,23}$. The 2014 WHO report on Implementation of Option B + for PMTCT suggests that the Malawi experience has shown that underutilization of data exists in the health facilities due to the HMIS approach that does not actively engage those at the sub-district level in data analysis and use ${ }^{8}$. These findings formulate the basis of claim that health workers at facility level provide clinical services with limited evidence based practice. This finding is supported by other limiting factors that exist in the health facilities: lack of information culture, low training, heavy workload, low education levels and inadequate data infrastructure. Similarly, studies have shown that most clinical practices are based on limited evidence and the latter factors hinder evidence based practice ${ }^{24-31}$. In addition, the ultimate success of a health information system is measured by informed decisions that lead to action and positive change in the health system or health status, rather than by the quantity or quality of data produced ${ }^{32}$. Therefore, HMIS approach needs to include facility level in the processes of data analysis and interpretation to increase data utilization and address the limiting factors.

The findings also suggest that health workers' perception toward the nature of the system contributes to the performance of PMTCT IM activities. The results showed that health workers perceived IT systems to be valuable and beneficial compared to paper-based systems that were perceived as tedious, error-prone, expensive, and difficult to manage. This finding is also consistent with a study conducted at Nathenje Health Centre which concluded that paper-based system is not a preferred method for health workers ${ }^{33}$. Therefore, if users have a negative attitude toward the system, their willingness to use the system effectively is questionable. In our study, the PMTCT health workers had negative attitudes toward the paper-based system, which could have led to PMTCT IM tasks being conducted without diligence, compromising data quality. IT tools provide an opportunity for enhanced performance on information management tasks that can improve data quality and use. Thus the government and its partners need to continue addressing the following limitations: inadequate knowledge or technical know-how, limited accessibility and lack of maintenance for improved utilization of IT tools.

\section{Limitations of the study}

The qualitative data were limited to the information that the participants have provided in the interviews and they might have had reservations in providing their true perceptions of the PMTCT IM at the facility. Additionally, the study was cross-sectional, conducted in only 5 out of forty-three health facilities in Lilongwe district. Therefore, the findings may not necessarily be generalizable to other health facilities in the country.

\section{Conclusion}

Based on the study findings, there is need to change health workers' perception and find out ways of motivating data quality improvement practices in the health facilities, as health workers view this as a tangential, non-essential part of their job. Therefore, health facility leadership needs to promote information culture through enforcement of meetings, supervision and provision of feedback. This can also assist to enhance utilization of PMTCT data in the health facilities. In addition, the government and its partners should continue rolling out and enhancing competence of health workers on IT tools employed in the health facilities.

\section{Acknowledgement}

The authors are grateful for contributions by Lilongwe District Health Office and participating health facilities and study participants.

Wiza Kumwenda was funded by Fogarty AIDS International Training and Research Program and a 2010 development grant from the University of North Carolina at Chapel Hill Center for AIDS research.

Nora Rosenberg was supported by the Fogarty International Center of the National Institutes of Health under Award Number NIMH K99MH104154-01A1 and the Center for AIDS Research P30 AI50410.

\section{References}

1. Attawell K. Scaling Up Prevention of Mother To Child Transmission of HIV. Tearfund; 2008.

2. MoH Malawi. Prevention of Mother to Child Transmission of HIV a five year scale plan. Second. Malawi: Ministry of Health Malawi; 2008.

3. Gourlay A, Wringe A, Michael D, Reniers G, Urassa M, Todd J, et al. Challenges with routine data sources for PMTCT program monitoring in East Africa: insights from Tanzania. Glob. Health Action 2015;8

4. Kamanga E, Mofolo I, Martinson F, Mwale G, Murotho J, Mhango $\mathrm{J}$, et al. PMTCT Scale up: experience in technical assistance and mentorship. In Mexico City: XVII International AIDS Conference; 2008. p. 1 .

5. Chinkonde-Nkhoma JR, Hosseinpour M, Mofolo I, Moses A, Mtande T, Kachingwe M, et al. A Review of the "Option B Plus" roll-out in five districts of Malawi. In Washington D.C., USA; 2012.

6. Government of Malawi, Ministry of Health. Intergrated HIV Program Report (October - December 2014). Lilongwe, Malawi: Ministry of Health; 2014.

7. Schouten EJ, John A, Midiani D, Makombe SD, Mnthambala A, Chirwa Z, et al. Prevention of mother-to-child transmission of HIV and 
the health-related Millennium Development Goals: time for a public health approach. Lancet. 2011;378(282-84).

8. WHO. Implementation of Option B + for Prevention of Mother-ToChild Transmission of HIV: The Malawi Experience. Regional Office for Africa, Republic of Congo: WHO; 2014.

9. Schabetsberger T, Ammenwerth E, Andreatta S, Grati G, Haux R, Lechleitner G, et al. From a paper based transmission of discharge summaries to electronic communications in healthcare regions. Int $\mathrm{J}$ Med Inform. 2006;75:209-15.

10. Douglas GP, Gadabu OJ, Joukes S, Mumba S, McKay MV, BenSmith A, et al. Using Touchscreen Electronic Medical Record Systems to Support and Monitor National Scale-Up of Antiretroviral Therapy in Malawi. PLoS Med. 2010;7(8):6.

11. Rondinelli I, Bouchet B, And Rimal N, Assessment of the Quality of HIV/AIDS services in Malawi. Malawi: USAID Healthcare Improvement Project. 2011.

12. WHO. Joint WHO-UNICEF-UNAIDS-PEPFAR Capacity Building Workshop: Strengthening reporting and monitoring in the health sector for the African region. Johannesburg, South Africa; 27-30 September 2010.

13. Woelk G, Berhan AY, Kudiabor K, Mukaminega M, On'gech J, Nyirabihizi E, et al. A secondary analysis of retention across the PMTCT cascade in selected countries: Rwanda, Malawi, Kenya, and Swaziland. Washington D.C.: USAID; 2015.

14. Mate KS, Bennett B, Mphatswe W, Barker P, Rollins N. Challenges of routine health system data management in a large public program to prevent mother-to-child transmission in South Africa. PLOS One; 2009;4(5). https://doi.org/10.1371

15. Chaulagai C, Moyo C, Koot J, Moyo H, Sambankusi T, Khunga F, et al. Design and Implementation of health management information system in Malawi: issues, innovations and results. Oxf Univ Press Assoc Lond Sch Hyg Trop Med. 2005;20(6):375-84.

16. AHMAC. National Health Information Management Principle Committee: Strategic Work Plan 2007-08 to 2012-12. Australian Health Ministers' Advisory Council; 2007.

17. Rad AM, Shams G, Naderi B. Information Management and the Role of Information and Knowledge Managers: Managers Perspectives. In Barcelona, Spain; 2009.

18. Khomani P, Mputeni C, and Gadabu O. Use of Electronic Medical Records System to Enforce Adherence to Maternal and Infant Health Treatment Protocol in Malawi: A Pilot Case Study at Bwaila Maternity Hospital. UbuntuNet-Connect Transforming Education and Research, 14 - 15 November 2013, Lemigo Hotel, Kigali Rwanda

19. RHINO. The RHINO Workshop on Issues and Innovation in Routine Health Information in Developing Countries. In Potomac, MD,
USA: Routine Health Information Network; 2001.

20. Lungo JH. Data Flows in Health Information Systems [Master Thesis]. University of Olso; 2003.

21. Kimaro HC, Twaakyondo HM. Analysing the hindrance to the use of information and technology for improving efficiency of healthcare delivery system in Tanzania. Tanzan Health Res. 2005;7.

22. Moyo C, Kaasbøll J, Nielsen P and Sæbø J. The Information Transparency Effects of Introducing League Tables in The Health System In Malawi, Electronic Journal of Health Information Systems in Developing countries.2016 75(2).

23. Republic of Malawi, Health Metric Network. Health Information System Assessment Report. Lilongwe, Malawi: MoH Malawi; 2009.

24. Booth A, Brice A. Evidence Based Practice for Information Professionals: A Handbook. London: Facet Publishing; 2004. 320 p.

25. Alspach G. Nurses' Use and Understanding of Evidence-Based Practice: Some Preliminary Evidence. Am J Crit Care. 2006;26(6):11-2.

26. Penz KL, Bassendowski SL. Evidence Based Nursing in Clinical Practice: Implications for Nurse Educators. J Contin Educ Nurs. 2006;37(6).

27. Beyea SC, Slattery MJ. Evidence Based Practice in Nursing: a guide to success implementation. HCPro; 2006.

28. Wall S. A critique of Evidence Based Practice in Nursing: Challenging the Assumptions. Palgrave Macmillan Ltd. 2008;6:37-53.

29. Jennings ET, Hall JL. Evidence Based Practice and the use of information in state agency decision making. In University of Kentucky, Lexington, Kentucky: Institute for Federalism and Intergovernmental Relations; 2009.

30. Nguyen TA, Oosterhoff P, Pham YN, Hardon A, Wright P. Health workers' Views on Quality of Prevention of Mother-to-Child Transmission and Postnatal Care for HIV-Infected Women and their Children. Hum Resour Health. 2009;39(7).

31. Majid S, Foo S, Luyt B, Zhang X, Theng Y, Chang Y, et al. Adopting evidence based practice in clinical decision making: nurses' perceptions. J Med Libr Assoc. 2011;

32. Land FF, Kennedy-McGregory M. Effective Use of Internal Medicine. London: London School of Economics and Political Science; 2002. Report No. 119.

33. Msukwa MKB. User Perceptions on Electronic Medical Record System (EMR) in Malawi. [College of Medicine]: University of Malawi; 2011. 